\title{
Corrigendum to: "Ultrasound Guided Wire Localization of Anal Tract in Imperforate Anus"
}

\author{
Seyed Ali Alamdaran (iD ${ }^{1}$, Jahanbakhsh Hashemi ${ }^{2}$, Ali Feyzi ${ }^{2}$, Ali Azadmand ${ }^{3}$, Masoud Mahdavi \\ Rashed $^{2}$, Samira Jafari ${ }^{2}$ and Marjan Joodi ${ }^{*}, 1$ \\ ${ }^{1}$ Surgical Oncology Research Center, Mashhad University of Medical Sciences, Mashhad, IR Iran \\ ${ }^{2}$ Department Radiology, Mashhad University of Medical Sciences, Mashhad, IR Iran \\ ${ }^{3}$ Faculty of Medicine, Mashhad University of Medical Sciences, Mashhad, IR Iran \\ "Corresponding author: Surgical Oncology Research Center, Mashhad University of Medical Sciences, Mashhad, IR Iran. Tel: +98-9155031236, Email: joodim@mums.ac.ir
}

Received 2019 December 31; Accepted 2020 April 24.

This corrects the article "Ultrasound Guided Wire Localization of Anal Tract in Imperforate Anus".

We have completed the study entitled "Ultrasound Guided Wire Localization of Anal Tract in Imperforate Anus" by Alamdaran et al. published in the Iran J Pediatr 2018; 28(4): e65338 (1). We would like to add some commentary to it.

Continence in patients with imperforate anus is related to exact anus reconstruction from middle of sphincter complex. We designed this study with purpose to investigate the ability of ultrasound in detection of the location and pathway of sphincter muscle complex in children with Imperforate Anus.

Our ultrasound findings in this study included (1) concentric multi-layered structures in subcutis (probably anal pit). (2) Concentric multi-layered structures in depth of pelvic floor (probably anal sphincter complex). (3) Difference in distance of rectal pouch and the surface of skin through probably anal pit and anal sphincter complex pathway with the shortest distance. (4) The ability to differentiate the types of internal fistula.

We also found four sonographic diagnostic pitfalls in these patients:

1. Difficulty of differentiation of muscle complex sphincter from other tissues by ultrasound; in adult, hypoechoic muscles is completely differentiable from echogenic fat, but in infants, iso-echogenicity of muscle sphincter complex with perineal fat is problematic and questionable. In addition, decreasing resolution of ultrasound images with increasing depth cause poor visualization of deep muscle sphincter, especially in high type.

2. Similarity of appearance of muscle sphincter complex with ectopic rectal pouch of the high type IA with perineal fistula mimics the gut signature appearance of concentric multi-layered structures and may be wrongly mistaken for low type of anomaly.
3. In literature, there are various cut offs for distance between rectal pouch and the surface of skin: some diagnostic cut off overlaps from $5 \mathrm{~mm}$ until $25 \mathrm{~mm}$ to determine the level of anorectal malformations (low and high). In addition, different measurement methods (the shortest distance or the distance obtained using anal sphincter complex pathway) render different distances (2-6).

4. Pressure of ultrasound probe or saline infusion through distal colostomy was the cause of underestimating the distance between rectal pouch and the surface of skin. The surgical distance is longer than the ultrasound distance.

Although these ultrasound findings could help to determine the level and type of disorder in order to plan and select the less invasive surgical technique, even to choose image guided surgery (6), it has a number of diagnostic pitfalls that can lead to serious diagnostic error. For better understanding these sonographic findings and decreasing of pitfalls further research is proposed.

\section{Acknowledgments}

The authors gratefully acknowledge the contribution of Dr. Shojaeean R and Dr. Parvizi MM for supporting us to conduct this study

\section{Footnotes}

Authors' Contribution: Study concept and design: Alamdaran SA and Joodi M; analysis and interpretation of data: Alamdaran SA and Joodi M; drafting of the manuscript: Alamdaran SA; critical revision of the manuscript for important intellectual content: Alamdaran SA and Joodi M.

Conflict of Interests: None. 
Ethical Approval: Ir.mums.fm.rec.1386.131.

Funding/Support: None.

Informed Consent: Written informed consent was obtained from the neonates' parents.

\section{References}

1. Alamdaran SA, Hashemi J, Feyzi A, Azadmand A, Mahdavi Rashed M, Jafari S, et al. Ultrasound Guided Wire Localization of Anal Tract in Imperforate Anus. Iranian Journal of Pediatrics. 2018;28(4). e65338. doi: 10.5812/ijp.65338.

2. Alamdaran SA, Joudi M, Azadmand A, Sabzevari A, Abdollahi A, Feyzi A, et al. Preoperative Trans-Perineal Sonographic Findings in Children with Imperforate Anus for Detection of Anal Sphincter Muscle Com- plex and the Anal Canal Pathway: A Pilot Study. Iranian Journal of Radiology. 2018;15(3). doi:10.5812/iranjradiol.57944.

3. Choi YH, Kim IO, Cheon JE, Kim WS, Yeon KM. Imperforate anus: determination of type using transperineal ultrasonography. Korean J Radiol. 2009;10(4):355-60. doi: 10.3348/kjr.2009.10.4.355. [PubMed: 19568463]. [PubMed Central: PMC2702044].

4. Ale HM, Molaeian M, Salamati P, Pourang H, Sedighi A. Validity of transperineal ultrasound in infants with imperforate anus. Iranian Journal of Radiology. 2004;2(1-2):43-7.

5. Nemati M, Oskouei RA, Aslanabadi S. Assessment of diagnostic value of transperineal ultrasonography in determining type of imperforate anus. J Am Sci. 2013;9(10s):91-5.

6. Haber HP, Seitz G, Warmann SW, Fuchs J. Transperineal sonography for determination of the type of imperforate anus. AJR Am J Roentgenol. 2007;189(6):1525-9. doi: 10.2214/AJR.07.2468. [PubMed: 18029895]. 